\title{
Género y política en los procesos electorales La participación política de la mujer como candidata y electa en las elecciones autonómicas y locales de 1995 en Canarias
}

\author{
Fermín Romero Navarro \\ Universidad de Las Palmas de Gran Canaria \\ Departamento de Psicología y Sociología \\ C/ Sta. Juana de Arco, 1. 35004 Las Palmas de Gran Canaria
}

Data de recepció: novembre 2000

Data d'acceptació: abril 2001

\section{Resumen}

En este artículo se presenta un estudio descriptivo de la presencia femenina en las listas electorales de las elecciones autonómicas y locales de 1995 y, como consecuencia de éstas, en las instituciones políticas de las Islas Canarias durante la legislatura 1995-1999. Atendiendo a los porcentajes de mujeres como candidata y electa, su distribución en las listas electorales, los cargos políticos ocupados, su diferente presencia según el tipo de institución y la dimensión de la circunscripción, el autor detecta dos mecanismos principales de exclusión de la mujer: la lejanía institucional y los puestos de salida, mientras que el carácter urbano o rural de la circunscripción tiene un efecto de menor consideración.

Palabras clave: mujer, género y política, procesos de reclutamiento de elites políticas.

\section{Abstract. Gender and politics in the electoral processes}

This paper presents a descriptive survey on the female presence in electoral rolls for the 1995 regional and local elections in the Canary Islands, and as elected members of the canarian political institutions during the 1995-1999 term. Regarding women's percentages as candidattes and elects, their distribution within electoral rolls, their different wheight in the three institutional levels analized (local, insular, regional), and the different population levels of the electoral districts, the author detects two main mechanismes of exclusion of women: institutional distance and the position in electoral rolls, while the urban or rural character of the different districts seems not to have any decisive role to play.

Key words: woman, gender and politics, political elite recruitment processes.

\section{Sumario}

1. Introducción 4. Conclusiones

2. Marco teórico Bibliografía 


\section{Introducción}

El presente artículo se entiende como parte de la corriente investigadora actual dedicada al seguimiento de los cambios en el sistema de género en las sociedades avanzadas y, en el caso que nos ocupa, de la incorporación de la mujer a la política. Concretamente, este estudio se acerca a la realidad de la presencia femenina en el poder político-institucional de las Islas Canarias a través de la investigación de su presencia en las listas electorales y en las instituciones políticas.

Este estudio tiene un carácter regional: se pretende ofrecer una imagen de la realidad política de la mujer en una sociedad concreta, con sus condicionantes concretos, lo que limita el alcance del estudio, pero también aumenta su fiabilidad. El carácter del estudio es descriptivo, atendiendo a una serie de motivaciones: no existen prácticamente estudios previos sobre el sistema de género y política en Canarias ${ }^{1}$, por lo que éste sería uno de los pioneros; se pretende obtener información básica que pueda servir de fundamento o complemento para posteriores investigaciones del mismo tipo, estableciendo series temporales, encuestas o trabajos cualitativos; al mismo tiempo se persigue cubrir una laguna investigadora: las Islas Canarias son territorio virgen para la sociología del género, contándose apenas un puñado de investigadores, mientras que los estudios nacionales apenas recogen unos pocos casos de las Canarias en sus muestras, por lo que la representatividad es francamente insuficiente.

La investigación se plantea longitudinalmente desde 1979 hasta 1995 para Gran Canaria, disponiéndose de dos bases de datos distintas pero complementarias: de 1979 a 1991, datos electorales de todas las instituciones en la isla de Gran Canaria y, para 1995, datos electorales regionales, comprendiendo todas las islas y todas las instituciones.

Como primer acercamiento al tema y a la complejidad de los procesos en él implicados, sirvan tres datos tomados a vuelo de pluma:

- Pimero: comparando los resultados de las elecciones autonómicas y locales de 1995 en Canarias con los de las anteriores convocatorias, se observa un ascenso continuado de la participación femenina en las categorías de candidata y elegida en todas las insituciones políticas: ayuntamientos, cabildos y parlamento autonómico.

- Segundo: en términos porcentuales se dan mayores índices de participación de la mujer como candidata al parlamento autonómico en todos los comicios habidos desde 1983. Esta participación no sólo aumenta de convocatoria en convocatoria, sino que es superior a la observada en las otras insituciones.

- Tercero: contrariamente a lo anteriormente expuesto, la participación de la mujer entre los elegidos al Parlamento de Canarias es la menor de todas las instituciones.

1. Con la notable excepción de Molina PeTiT, C. (1996). La igualdad no resuelta. Mujer y participación política. Un estudio en Gran Canaria. Las Palmas de Gran Canaria: Instituto Canario de la Mujer. 
Estos datos nos dan la imagen positiva de un aumento de la presencia femenina y la negativa de una persistencia de la exclusión de la mujer en los procesos políticos. La complejidad del tema y la carencia de fuentes que permitan análisis e interpretaciones nos animan a presentar este estudio, que pretende cubrir los aspectos que detallamos a continuación.

\subsection{Objetivos}

El presente estudio tiene los tres objetivos siguientes:

1. Estudio descriptivo, de carácter longitudinal, de la participación política de la mujer como candidata y elegida en los comicios electorales celebrados en Canarias durante la presente etapa democrática.

2 Determinación de la influencia del factor género en el posicionamiento de las mujeres en las listas electorales y, en su caso, en las responsabilidades de gobierno, ponderando el papel de otras variables como el tipo de institución, factores territoriales y los puestos, en lista.

3. Determinación del papel desempeñado por los distintos mecanismos de exclusión.

\subsection{Método}

El estudio se realiza partiendo de la combinación de las dos bases de datos antes mencionadas. El conjunto de datos así obtenido se refiere a la medición cuantitativa de la participación de hombres y mujeres en la totalidad de comicios celebrados en Canarias entre 1979-1991 (Gran Canaria) y 1995 para la Comunidad Autónoma en las categorías de candidatos y elegidos para todas las instituciones y en todas las circunscripciones. Dichos datos provienen de los boletines oficiales de las provincias de Las Palmas de Gran Canaria y Santa Cruz de Tenerife, el Boletín Oficial de Canarias y los datos electorales publicados en la prensa local del archipiélago.

Las variables que queremos medir son la proporción de hombres y mujeres en las categorías de candidatos y elegidos, así como su situación en las listas electorales en función de la institución, el tipo de circunscripción y el partido político.

La recogida de datos se realizó en dos etapas. La primera etapa se centró, durante el año 1995, previamente a las elecciones, en la recogida de los documentos relacionados con la composición de las candidaturas a las distintas instituciones. Posteriormente a las elecciones, en una segunda etapa, se recopilaron los documentos que recogían las listas de los elegidos.

Realizada esta fase de recolección de datos, se pasó a su tratamiento y clasificación, procediendo a su vaciado en plantillas por circunscripciones (municipio o isla) e instituciones (ayuntamientos, cabildos insulares, Parlamento autonómico), continuando esta tarea con la agregación de los datos de cada circunscripción a los niveles insular, provincial y autonómico. 
Finalmente, todo este material fue informatizado y sometido a tratamiento estadístico, usando principalmente las medidas de concentración. Un primer resultado de esta tarea fue la descripción pormenorizada de las candidaturas presentadas y elegidas en todos y cada uno de los municipios de la Comunidad Autónoma de Canarias, incluyendo la posición en lista, la pertenencia a partidos políticos y el tipo de municipio por isla y magnitud poblacional.

La medición de los datos electorales de los anteriores comicios (1979-1991) en Gran Canaria se tomó de anteriores estudios del autor ${ }^{2}$.

\section{Marco teórico}

La incorporación de la mujer a los ámbitos del poder político y, más concretamente, como candidata y electa en los comicios electorales en la Comunidad Canaria, necesita de un marco de comprensión teórica desde donde valorar el fenómeno en cuestión.

Los tres aspectos que vamos a desarrollar en este marco teórico son: el sistema de género y política; entre la igualdad formal y la igualdad real de la mujer en los espacios sociales intermedios, y la mujer como agente de cambio y sujeto político.

\subsection{El sistema de género y politica}

La variable género continúa teniendo una gran importancia para la comprensión del comportamiento diferencial de los ciudadanos con relación a la política, ya sea con respecto a los rasgos de la cultura política o a la participación electoral y en organizaciones políticas.

En relación con las diferencias de posicionamiento y la participación política de las mujeres, existían dos tipos de explicaciones ya superadas, una por errónea y otra por insuficiente. La una, propia de los conservadores, considera que las diferencias de la mujer en la participación política vienen dadas por las características biológicas y/o por sus rasgos psicológicos. Es decir, las características físicas femeninas influencian sus comportamientos y determinan sus intereses políticos. La otra, propia de los progresistas, argumenta que son los factores sociales, que se transmiten a través de la educación, los que explican las diferencias políticas de las mujeres con respecto a los varones.

El sistema género, es decir, la división sexual del trabajo, y la organización social que lo regula, la cultura patriarcal, aún vigente, aunque atenuada, es asumida como un paradigma sin el cual se hace casi imposible explicar las diferencias entre las mujeres y los varones, en relación con la participación política. Este nuevo enfoque se presenta como un sistema de análisis alternativo a las explicaciones tradicionales antes expuestas.

2. Cf. Romero Navarro, F. (1996). La liberación de la mujer en Gran Canaria. Cambio en la estructura familiar. Las Palmas de Gran Canaria: Universidad de las Palmas de Gran Canaria, Servicio de Publicaciones. 
¿Qué aporta esta perspectiva al tema que nos trae? Nos aporta una doble aproximación, lo que explicaremos siguiendo el pensamiento de Judith Astelarra ${ }^{3}$.

\section{Primero: la política está determinada por el sistema género}

El espacio político es conformado como un espacio masculino. Las actividades políticas y sus organizaciones no se constituyen al margen de la división sexual del trabajo, sino que vienen determinadas por las formas diferenciadas que tienen las mujeres y los varones de desarrollar las actividades sociales.

La división sexual moderna del trabajo se consolida con la industrialización y el capitalismo. La vida social, contrariamente a la uniformidad que existía en las sociedades anteriores a la industrialización, se divide en dos ámbitos claramente diferenciados: la vida privada y la vida pública. A la familia se le atribuían las tareas relacionadas con la reproducción humana y el cuidado de los hijos, siendo las mujeres las responsables de las mismas, y la economía, la política y la cultura se convirtieron en tareas públicas asignadas a los varones. La producción de bienes se trasladó a las fábricas y se restringió el concepto de trabajo para referirse sólo al trabajo productivo que recibe un salario, una retribución económica.

La acentuación de la separación entre ambas esferas sociales, lo privado y lo público, produjo una radicalización de la división sexual del trabajo, lo que a su vez ha generado, entre otros, dos tipos de limitaciones en contra de la participación de la mujer en las instituciones políticas:

a) Tiempo disponible: al atribuir a las mujeres las tareas domésticas y educativas de los hijos como responsabilidades primordiales y exclusivas, se les deja menos tiempo que los varones para intervenir en la "cosa pública». Es cierto que hay mujeres que lo hacen, pero es a costa de emplear la llamada "doble jornada». Sus esfuerzos son mayores que los de sus colegas los varones. A lo indicado hay que sumar otras desventajas de carácter cultural que se derivan de la socialización en los patrones de la "psicología femenina», según la cual a las mujeres se les veta para no asumir deseos ni comportamientos propios del competir y la agresividad de la lucha política.

b) Reproducción por parte de las mujeres del esquema materno-familiar en espacios políticos. Se constata que las mujeres que acceden a las instituciones de gobierno lo hacen en aquellos sectores o áreas en los que, de alguna forma, prolongan sus actividades de protección y cuidado familiar, como son las áreas sociales, culturales, educativas y medioambientales, pero muy escasamente desarrollan las actividades de comercio, hacienda, industria, defensa, etc. Este modelo parece indicar una cierta «concesión» por parte del poder masculino para que la mujer participe en algunas áreas del poder político y no en otras.

3. Astelarra, J. (1990). «Las mujeres y la política». En CIS. Participación política de las mujeres. Madrid: Siglo XXI, p. 7-22. 


\section{Segundo: la relación entre socialización familiar y política}

A pesar de la pérdida de funciones que ha experimentado, la familia nuclear sigue siendo el primer y más importante agente de socialización, sobre todo en patrones normativos básicos que estructuran la personalidad futura de los hijos. Este poder socializador se extiende también al orden político, en cuanto que la familia se convierte en conformadora de opiniones, actitudes y decisiones, como tradicionalmente suceden en los procesos electorales.

La familia tiene una influencia decisiva en la configuración del comportamiento político de los individuos, en cuanto que es también una organización social básica, donde existen relaciones políticas, entendidas como relaciones de poder. Este poder lo ha sustentado el varón, encarnado en la figura del padre. Este poder tiene una dimensión económica, es decir, se basa en la propiedad de la hacienda familiar del pater familiae de ayer o en el trabajo e ingresos del esposo e hijos varones de hoy, todo lo cual genera una desigualdad y dependencia en las relaciones con el varón. Es cierto que este esquema va cambiando debido, entre otros factores, a la incorporación de la mujer al trabajo, pero el referente simbólico del varón como aglutinador del poder económico y fuente de poder permanece y estructura la vida social de la familia.

De lo expuesto se deduce un corolario: la familia es una institución social básica que tiene la capacidad de socializar en la discriminación del poder, ya sea porque legitima el poder masculino, o porque socializa desigualmente a los niños y a las niñas en las relaciones de poder, señalando a los unos su espacio en el mundo público y a las otras que su ámbito de desarrollo principal está en la vida privada, conformada por el mundo doméstico.

\subsection{Entre la igualdad formal y la igualdad real de la mujer:} los espacios sociales intermedios

La igualdad formal de la que goza la mujer en las sociedades desarrolladas se sitúa en un proceso histórico, más que secular, que va desde los movimientos sufragistas hasta la actual conquista de los llamados «derechos sociales». Como tal proceso democrático se inserta dentro de las grandes transformaciones económicas, laborales, educativas, culturales, sanitarias y tecnológicas que se ponen en marcha a partir de y a impulso de la revolución industrial.

El reconocimiento formal de la dignidad humana, expresado en la Declaración Universal de los Derechos Humanos, se sitúa todavía más en el orden del «debe ser», la meta que inspira y a la que debe aspirar el ordenamiento jurídico-social, que en el orden de la realidad social, que se mueve no tanto a impulsos de la conciencia reflexiva como a impulsos de los intereses socioeconómicos y culturales, siempre contrapuestos. Estamos entre el «sí» del reconocimiento de los derechos de la mujer y el «todavía no».

¿Por qué «todavía no» a la participación política de la mujer en igualdad de condiciones con el varón? 
Existen múltiples y variados espacios sociales en los que los cambios ideológicos y culturales favorables a la igualdad entre el varón y la mujer se producen por vías lentas y tantas veces contradictorias.

El matrimonio y las relaciones familiares; el trabajo asalariado de la mujer; el acceso y el desempeño del poder, sea público o privado, y las concepciones culturales en torno a los roles del varón y la mujer, son espacios que poseen sus propias singularidades y sus propios ritmos respecto a la consideración de la igualdad real de los derechos de la mujer.

Alcanzar mayores cotas de poder por parte de la mujer en los ámbitos del poder político es un propósito de todos los partidos y gobiernos democráticos. Para alcanzar este objetivo, unos proponen la implantación de una cuota femenina, la llamada "discriminación positiva», como es el caso del PSOE e IU; otros consideran que son las propias capacidades de las mujeres las que les harán valedoras para el puesto en la lista electoral o el cargo público, como es el caso del PP. Sin embargo, las últimas elecciones de 1999 a los parlamentos autonómicos y al Parlamento Europeo y las recientes elecciones del 12 de marzo de 2000 al Congreso y al Senado no han alcanzado todavía un tercio de mujeres electas, situándose los resultados en los siguientes valores: para las comunidades autónomas, el 28,79\%, siendo Castilla-La Mancha y la Comunidad Valenciana las que mayores porcentajes femeninos alcanzan, con un $40,43 \%$ y un $40,45 \%$ respectivamente, y para el Parlamento Europeo el 29,71\%, siendo Finlandia, con un $43,75 \%$, y Francia y Suecia, con un $40,23 \%$ y un $40,11 \%$ respectivamente, los que alcanzan mayores puntuaciones. España tiene un $34,38 \%$ de mujeres parlamentarias europeas, 22 respecto de 64 escaños. En cuanto a las elecciones recientes del 12 de marzo, las mujeres alcanzan el $28 \%$ en el Congreso, lo que supone un aumento de seis puntos con relación a las elecciones de 1996, y en el Senado logran el 25\%, dándose también un aumento respecto a 1996 de 11,84 puntos porcentuales ${ }^{4}$. ¿Cómo se explica esta diferencia con los varones?

La batalla de la participación política de la mujer se libra previamente en los espacios intermedios como son la familia, los niveles educativo-profesionales, la incorporación al mercado de trabajo y los cambios culturales respecto a la concepción y roles del varón y de la mujer.

La incorporación de la mujer a la educación media y superior, al trabajo remunerado en general y sobre todo al trabajo profesional cualificado son factores que cuestionan el sistema de roles específicos de género. La desigual distribución de las responsabilidades familiares y sociales en función del género, que aún permanece, actúa de freno para que la mujer pueda acceder, permanecer, competir y ascender en pie de igualdad con los varones, tanto en el mercado de trabajo como en los puestos directivos de carácter económico y político. La mujer necesita y quiere conciliar su mundo afectivo-familiar con

4. Cf. Instituto de la Mujer (2000). Resultados electorales. Madrid: Ministerio de Trabajo y Asuntos Sociales. 
estos espacios. Para lograr esta conciliación quedan pendientes conquistas reales encaminadas a diseñar mejores y más asequibles sistemas de cuidado de los niños y personas dependientes, más guarderías con ampliación de horarios, extensión de la educación infantil, mejora del sistema del permiso paterno, adaptación de los horarios comerciales y de otros servicios, así como el establecimiento de políticas de empleo favorecedoras de la conciliación entre el ámbito laboral y el familiar. Éstos son los espacios sociales intermedios donde se libra la batalla cotidiana por la igualdad de las mujeres.

Los mayores niveles educativos que están alcanzando las mujeres en todas las carreras universitarias, el descenso del número de hijos, lo que a su vez es una amenaza para el reemplazo generacional y el sostenimiento de los sectores pasivos y, en cierta medida, el auge del ciclo económico, son factores que incentivan la entrada de mano de obra femenina al mercado laboral, lo que no significa conseguir un trabajo ni que éste sea cualificado, ni estable, ni a tiempo completo. El desempleo femenino es mayor que el de los varones en todos los países de la Unión Europea. En España, donde se da la mayor tasa de desempleo global de los países de la UE, es todavía mayor para las mujeres, alcanzando la puntuación del 26,8\%.

La segregación negativa de los colectivos de mujeres trabajadoras pasa por el trabajo a tiempo parcial, como se desprende de los siguientes datos: de todas las trabajadoras de la UE un tercio trabaja menos de las 35 o 40 horas oficiales, circunstancia que afecta sólo a un 5\% de los varones europeos, que por lo general son estudiantes o mayores prejubilados ${ }^{5}$. Esta segregación se extiende también a los distintos sectores de producción en desventaja para la mujer, empleándose sobre todo en el comercio, y principalmente en ventas. Realizan mayoritariamente trabajos administrativos, servicios de medicina, enfermería, enseñanza, cuidados a terceros, todo lo cual lleva consigo la ocupación de oficios de menor rango y remuneración y con menos probabilidades de promoción que las ocupaciones de los varones.

La segregación laboral de las mujeres alcanza también a los niveles retributivos, aunque las diferencias tienden a disminuir. Según los estudios de Montiel Torres antes citados, en 1997 las ganancias por hora de las mujeres como porcentaje en relación con la de los hombres eran: 84\% en Suecia, 73\% en Francia y España y hasta el 64\% en el Reino Unido.

La autonomía de la mujer es la condición básica para que pueda acceder a los ámbitos del poder social y político. Pero la autonomía personal de la mujer pasa necesariamente por la autonomía que le proporciona la consecución de la igualdad en todos los espacios sociales intermedios que hemos apuntado. 


\subsection{La mujer como agente de cambio y sujeto político}

La consideración de la mujer como agente de cambio y sujeto político puede ser entendida como una contradicción a los aspectos anteriormente expuestos, pero no es así. La realidad social no es uniforme ni lineal, es compleja, está sometida a influencias de factores múltiples cuyos efectos se convierten muchas veces en causas. Por todo ello adoptamos un enfoque teórico-explicativo de carácter circular, multicausal y no-lineal, a partir del cual se comprende el desarrollo del presente punto.

El siglo XX puede ser definido como el siglo de la conquista de los derechos formales y sociales de la mujer, aunque es cierto que se ha circunscrito más a las sociedades desarrolladas. Ha sido largo el camino histórico que ha tenido que recorrer la mujer para lograr la liberación, la emancipación y la promoción como persona en pie de igualdad con el varón, con la consecuente transformación de las estructuras sociales, no exenta de contradicciones y de un ritmo lento.

Este proceso ha venido impulsado por los diversos movimientos de emancipación y liberación de la mujer, prestando una triple función: de mediación con la sociedad y sus estructuras, de clarificación de la conciencia colectiva en torno al concepto o construcción de la mujer y de función de presión encaminada a acelerar el cambio.

Lo sorprendente de la acción de los movimientos feministas está en que éstos han desbordado sus propios límites y han puesto en marcha un proceso de cambio social de tal envergadura y trascendencia que las importantes transformaciones sociales actuales no se comprenden si no se considera a la mujer como sujeto activo de los mismos y por ello sujeto de cambio social.

La importancia de este proceso es de tal magnitud que, tras conseguir que valores fundamentales, negados por una cultura agraria-patriarcal, se conviertan en derechos positivos, a su vez se produzca una transformación de las concepciones tradicionales del varón y de la mujer, permeando cualquier rincón de la vida social, económica, política y religiosa.

La nueva conciencia que la mujer ha construido de sí misma le permite no sólo reivindicar la igualdad con el varón, sino que le posibilita equiparse de aquellos recursos personales, educativos, profesionales que la hace valedora y protagonista de su causa.

La mujer actual está pilotando el cambio social como un agente histórico de acción social en cualquiera de los espacios sociales tradicionalmente reservados al varón. Esta perspectiva nos permite comprender la importancia política, todavía limitada pero in crescendo, que está adquiriendo la mujer en los ámbitos del poder político. Ello nos permite comprender el cambio que al respecto se está produciendo: la mujer deja de ser un sector de votantes dirigido por la ideología de los varones y está pasando a ser un sector con personalidad propia, saliendo de entre sus filas mujeres líderes que quieren conseguir y ejercen el poder político. La presencia notable de mujeres en las 
primeras filas de las candidaturas de los partidos en las recientes elecciones a las Cortes Generales se ha convertido en un tirón electoral decisivo para ganar escaños.

\section{Análisis de los datos}

En primer lugar, procederemos al análisis de los datos electorales por instituciones, estudiando en cada una de ellas los datos agregados primero y, posteriormente, descendiendo a niveles territoriales inferiores, buscando sus particularidades y eventuales similitudes.

En segundo lugar, haremos referencia a las posibles similitudes y diferencias entre instituciones, buscando discernir la relevancia del factor territorial.

Previamente, se considera necesario exponer la evolución de la participación de la mujer en los comicios celebrados en Gran Canaria entre 1979 y 1991. Este estudio previo, circunscrito a la citada isla, motivó el presente estudio, sirviendo de referencia para conocer la evolución del fenómeno.

\subsection{Participación de la mujer en los comicios electorales celebrados en la isla de Gran Canaria (1979-1991)}

Las primeras elecciones democráticas a las instituciones locales tuvieron lugar en 1979, en este apartado se estudiarán las tres convocatorias siguientes. Las primeras elecciones autonómicas se convocaron en 1983; la última de las convocatorias estudiadas en este apartado es la de mayo de 1991. Con el fin de analizar la participación político-electoral de la mujer en la isla de Gran Canaria, procedemos a analizar el número de candidatos/as y elegidos/as a las instituciones locales y autonómicas según sexo y año de convocatoria. La observación de las tablas 1 y 2 nos permite realizar las siguientes consideraciones ${ }^{6}$.

Un primer dato significativo se refiere a la notablemente escasa presencia de la mujer, tanto en la condición de candidata como de elegida. Si contrastamos este dato con la presencia de los hombres, la situación es bastante reveladora: las diferencias de participación son muy llamativas, manteniéndose esta situación a lo largo de las cuatro legislaturas, aunque hay que reconocer un permanente ascenso, pero aún poco significativo.

Un segundo dato hace referencia a que esta mínima participación femenina se acentúa a medida que subimos de la Administración local a la autonó-

6. Los datos para este capítulo provienen de Romero Navarro, F. (1996). La liberación de la mujer en Gran Canaria. Cambio de la estructura familiar. Las Palmas de Gran Canaria: Universidad de Las Palmas de Gran Canaria, Servicio de Publicaciones. En dicho capítulo se analizan las listas de resultados electorales oficiales, publicadas en los boletines oficiales de la Provincia de Las Palmas, en los que aparecen consignados los nombres, según los partidos políticos, de los candidatos a todas y cada una de las elcciones democráticas celebradas entre 1979 y 1991, elecciones municipales, insulares y autonómicas, así como los informes de la prensa local. 
Tabla 1. Candidatos/as y elegidos/as a las instituciones locales y autonómica según año y sexo. Gran Canaria, 1979-1991.

\begin{tabular}{|c|c|c|c|c|c|c|c|c|c|c|c|c|}
\hline \multirow[b]{3}{*}{ Año } & \multicolumn{4}{|c|}{ Ayuntamientos } & \multicolumn{4}{|c|}{ Cabildo } & \multicolumn{4}{|c|}{ Parlamento autonómico } \\
\hline & \multicolumn{2}{|c|}{ Candidatos/as } & \multicolumn{2}{|c|}{ Elegidos/as } & \multicolumn{2}{|c|}{ Candidatos/as } & \multicolumn{2}{|c|}{ Elegidos/as } & \multicolumn{2}{|c|}{ Candidatos/as } & \multicolumn{2}{|c|}{ Elegidos/as } \\
\hline & $\mathrm{H}$ & M & $\mathrm{H}$ & M & $\mathrm{H}$ & M & $\mathrm{H}$ & M & $\mathrm{H}$ & M & $\mathrm{H}$ & M \\
\hline \multirow[t]{2}{*}{1979} & 1.569 & 216 & & 12 & & 10 & 27 & 0 & - & - & - & - \\
\hline & 87,9 & 12,1 & 96,4 & 3,5 & 97,7 & 7,3 & 100 & 0 & - & - & - & - \\
\hline \multirow[t]{2}{*}{1983} & 1.246 & 213 & 315 & 20 & & 45 & 25 & 2 & & & & 0,0 \\
\hline & 85,4 & 14,6 & 94,2 & 5,9 & 82,5 & 17,5 & 92,6 & 7,5 & 76,2 & 16,8 & 100 & 0,0 \\
\hline \multirow[t]{2}{*}{1987} & 2.355 & 488 & 327 & 22 & 321 & 81 & 26 & 1 & 198 & 52 & 15 & 0,0 \\
\hline & 82,8 & 17,2 & 93,6 & 6,3 & 79,8 & 20,2 & 96,3 & 3,7 & 79,2 & 20,8 & 100 & 0,0 \\
\hline \multirow[t]{2}{*}{1991} & 1.935 & 499 & 318 & 35 & 289 & 100 & 26 & 3 & 188 & 64 & 14 & 1 \\
\hline & 79,4 & 20,5 & 90,05 & 9,9 & 74,2 & 25,7 & 89,6 & 10,3 & 74,6 & 25,3 & 93,3 & 6,6 \\
\hline
\end{tabular}

Fuente: ROMERO NAVARRO, F., op. cit.

Tabla 2. Candidatos/as electos/as a las instituciones locales y autonómica. Gran Canaria, 1979-1991.

\begin{tabular}{|c|c|c|c|c|c|c|}
\hline \multirow[b]{2}{*}{ Año } & \multicolumn{2}{|c|}{ Ayuntamientos } & \multicolumn{2}{|c|}{ Cabildo } & \multicolumn{2}{|c|}{ Parlamento autonómico } \\
\hline & $\mathrm{H}$ & M & $\mathrm{H}$ & M & $\mathrm{H}$ & M \\
\hline 1979 & 325 & 12 & 27 & 0 & - & - \\
\hline 1983 & 315 & 20 & 25 & 2 & 15 & 0 \\
\hline 1987 & 327 & 22 & 26 & 1 & 15 & 0 \\
\hline 1991 & 318 & 35 & 26 & 3 & 14 & 1 \\
\hline
\end{tabular}

Fuente: Romero NAVARro, F., op. cit.

mica, es decir, que a medida que la esfera de la influencia política es más amplia, la mujer tiene una presencia menor, tanto como candidata como elegida. Esto es particularmente significativo en lo que respecta a las elecciones autonómicas, donde la presencia de la mujer como elegida es inexistente hasta 1991, año en que hace entrada en el Parlamento de Canarias la primera diputada regional.

Un tercer punto se refiere a que efectivamente se ha dado un aumento de la participación de las mujeres en cuanto a candidaturas propuestas, lo que, sin embargo, no se ha reflejado en una mayor participación en cuanto a cargos electos.

Esta situación analizada es el reflejo de las posiciones que ocupan las mujeres en las listas electorales en estas distintas instituciones.

Los porcentajes de mujeres que ocupan del primero al tercer puesto en las distintas candidaturas presentadas son muy reducidos, no excediendo en nin- 
gún caso del $9 \%$. Si tenemos en cuenta la distribución de los cargos públicos según los distintos partidos, con semejantes porcentajes, resulta bastante difícil la presencia significativa de mujeres en puestos de relevancia política.

Los lugares ocupados por la presencia de mujeres en las listas electorales se concentran en los tramos de posición del 10 al 30, como se observa en las tablas 3 a 5 .

A fin de sintetizar la información, se adjuntan cuadros que redundan en la participación de las mujeres en la actividad política canaria.

Tabla 3. Posición de las mujeres en las listas electorales según grupos. Gran Canaria, 1979-1991.

\begin{tabular}{|c|c|c|c|c|c|c|c|}
\hline \multirow[b]{3}{*}{ Año } & \multicolumn{7}{|c|}{ Ayuntamientos } \\
\hline & \multicolumn{5}{|c|}{ Grupos } & \multicolumn{2}{|c|}{ Suplentes } \\
\hline & $1-3$ & $4-10$ & $11-20$ & $21-30$ & $31+$ & $1-3$ & $4+$ \\
\hline \multirow[t]{2}{*}{1979} & 21 & 73 & 77 & 21 & - & 23 & 1 \\
\hline & 9,7 & 33,8 & 35,6 & 9,7 & - & 10,6 & 0,5 \\
\hline \multirow[t]{2}{*}{1983} & 16 & 61 & 88 & 41 & 1 & 5 & - \\
\hline & 7,5 & 28,6 & 41,3 & 19,2 & 0,4 & 2,4 & \\
\hline \multirow[t]{2}{*}{1987} & 40 & 135 & 1.556 & 61 & 5 & 53 & 38 \\
\hline & 6,4 & 29,6 & 37,2 & 11,4 & & 15,0 & 20,2 \\
\hline \multirow[t]{2}{*}{1991} & 32 & 148 & 186 & 57 & 0 & 75 & 1 \\
\hline & 6,4 & 29,6 & 37,2 & 11,4 & & 15,0 & 20,2 \\
\hline
\end{tabular}

Fuente: Romero NAVARRO, F., op. cit.

Tabla 4. Posición de las mujeres en las listas electorales. Gran Canaria, 1979-1991.

\begin{tabular}{|c|c|c|c|c|c|c|c|}
\hline \multirow[b]{3}{*}{ Año } & \multicolumn{7}{|c|}{ Cabildos } \\
\hline & \multicolumn{5}{|c|}{ Grupos } & \multicolumn{2}{|c|}{ Suplentes } \\
\hline & $1-3$ & 4-10 & $11-20$ & $21-30$ & $31+$ & $1-3$ & $4+$ \\
\hline \multirow[t]{2}{*}{1979} & - & 3 & 3 & 1 & - & 1 & 2 \\
\hline & & 30,0 & 30,0 & 10,0 & & 10,0 & 20,0 \\
\hline \multirow[t]{2}{*}{1983} & 1 & 8 & 12 & 21 & - & 3 & - \\
\hline & 2,2 & 17,8 & 26,7 & 46,7 & & 6,7 & \\
\hline \multirow[t]{2}{*}{1987} & 6 & 16 & 32 & 23 & 4 & - & - \\
\hline & 7,4 & 19,8 & 39,5 & 28,4 & 4,9 & & \\
\hline \multirow[t]{2}{*}{1991} & 2 & 22 & 37 & 33 & - & 6 & - \\
\hline & 2,0 & 22,0 & 37,0 & 33,0 & & 6,0 & \\
\hline
\end{tabular}

Fuente: Romero NAVArRo, F., op. cit. 
Tabla 5. Posición de las mujeres en las listas electorales. Gran Canaria, 1979-1991.

\begin{tabular}{lllllll}
\hline & \multicolumn{3}{l}{ Parlamento autonómico } & & \\
\cline { 2 - 3 } & \multicolumn{2}{l}{ Grupos } & & & Suplentes \\
\cline { 2 - 3 } Año & $\mathbf{1 - 3}$ & $\mathbf{4}-10$ & $\mathbf{1 1 - 2 0}$ & & $\mathbf{1 - 3}$ & $\mathbf{4}+$ \\
\hline 1983 & 3 & 10 & 14 & & 2 & - \\
& 10,3 & 34,4 & 48,2 & & 6,8 & \\
\hline 1987 & 5 & 21 & 16 & & 2 \\
& 9,6 & 40,4 & 30,8 & & 14,4 & 3,8 \\
\hline 1991 & 7 & 29 & 16 & & 12 & - \\
& 10,9 & 45,3 & 25,0 & & 18,8 & \\
\hline
\end{tabular}

Fuente: Romero NAVARro, F., op. cit.

\subsection{Las elecciones de 1995. Análisis según instituciones}

En este apartado procederemos al análisis de los datos referentes a la participación de la mujer como candidata y elegida. En el análisis de las candidaturas se compara la presencia de hombres y mujeres, su diferente distribución en las listas electorales según grupos y partidos y la ratio mujeres/hombres por grupos de posiciones en lista según los diferentes ámbitos territoriales e institucionales.

$\mathrm{El}$ análisis de los datos referentes a los candidatos elegidos se realiza atendiendo a los mismos criterios.

Por último se procede a la interpretación de los datos, tomando los correspondientes a las candidaturas como punto de partida y los de elegidos como resultados de los primeros.

En cuanto a la estructura del apartado, comenzamos por los ayuntamientos para terminar con el Parlamento de Canarias.

Para facilitar la comprensión del análisis, presentamos las tablas 6 y 7, en las que se refleja la presencia de varones y mujeres como candidatos/as y elegidos/as según circunscripciones e instituciones.

\subsubsection{Elecciones municipales de la Comunidad Canaria}

El 28 de mayo de 1995 concurrieron a las urnas 417 candidaturas con 8.029 candidatos repartidos entre los 89 municipios de que consta la Comunidad Autónoma de Canarias. De los 8.029 candidatos, 5.936 (73,93\%) eran hombres y $2.093(26,07 \%)$ mujeres con estas cifras queda de manifiesto la escasa participación femenina (tabla 8).

Como se desprende de la tabla 9 y del gráfico 1, existen dos tendencias, a saber:

- La proporción mujeres/hombres aumenta según se desciende en las listas electorales, alcanzando su máximo en los puestos inferiores al $21^{\circ}$ (donde los hay) y en los grupos de suplentes. 
Tabla 6. Candidatos/as y elegidos/as por circunscripciones. Canarias, 1995 (incluidas todas las instituciones).

\begin{tabular}{|c|c|c|c|c|c|c|c|c|c|c|c|c|}
\hline \multirow{3}{*}{$\begin{array}{l}\text { Todas las instituciones } \\
\text { canarias, } 1995\end{array}$} & \multicolumn{6}{|c|}{ Candidatos/as } & \multicolumn{6}{|c|}{ Elegidos/as } \\
\hline & \multicolumn{2}{|c|}{ Hombres } & \multicolumn{2}{|c|}{ Mujeres } & \multicolumn{2}{|c|}{ Total } & \multicolumn{2}{|c|}{ Hombres } & \multicolumn{2}{|c|}{ Mujeres } & \multicolumn{2}{|c|}{ Total } \\
\hline & $\mathrm{N}$ & $\%$ & $\mathrm{~N}$ & $\%$ & $\mathrm{~N}$ & $\%$ & $\mathrm{~N}$ & $\%$ & $\mathrm{~N}$ & $\%$ & $\mathrm{~N}$ & $\%$ \\
\hline Gran Canaria & 2.369 & 73,2 & 867 & 26,8 & 3.236 & 100,0 & 335 & 84,4 & 62 & 15,6 & 397 & 100,0 \\
\hline Lanzarote & 765 & 74,9 & 256 & 25,1 & 1.021 & 100,0 & 104 & 85,2 & 18 & 14,8 & 122 & 100,0 \\
\hline Fuerteventura & 458 & 74,6 & 156 & 25,4 & 614 & 100,0 & 87 & 88,8 & 11 & 11,2 & 98 & 100,0 \\
\hline Provincia de Las Palmas & 3.592 & 73,7 & 1.279 & 26,3 & 4.871 & 100,0 & 526 & 85,3 & 91 & 14,7 & 617 & 100,0 \\
\hline Tenerife & 2.463 & 72,6 & 931 & 27,4 & 3.394 & 100,0 & 433 & 82,2 & 94 & 17,8 & 527 & 100,0 \\
\hline La Palma & 724 & 74,1 & 253 & 25,9 & 977 & 100,0 & 167 & 83,1 & 34 & 16,9 & 201 & 100,0 \\
\hline La Gomera & 279 & 77,7 & 80 & 22,3 & 359 & 100,0 & 68 & 100,0 & 13 & 16,0 & 81 & 100,0 \\
\hline El Hierro & 192 & 73,8 & 68 & 26,2 & 260 & 100,0 & 31 & 86,1 & 5 & 13,9 & 36 & 100,0 \\
\hline Provincia de Santa Cruz & 3.658 & 73,3 & 1.332 & 26,7 & 4.990 & 100,0 & 699 & 82,7 & 146 & 17,3 & 845 & 100,0 \\
\hline Canarias & 7.250 & 73,5 & 2.611 & 26,5 & 9.861 & 100,0 & 1.225 & 83,8 & 237 & 16,2 & 1.462 & 100,0 \\
\hline
\end{tabular}

Fuente: elaboración propia.

Tabla 7. Candidatos/as y elegidos/as por instituciones y circunscripciones. Canarias, 1995.

\begin{tabular}{|c|c|c|c|c|c|c|c|c|c|c|c|c|}
\hline \multirow{3}{*}{$\begin{array}{l}\text { Elecciones } \\
\text { municipales }\end{array}$} & \multicolumn{6}{|c|}{ Candidatos/as } & \multicolumn{6}{|c|}{ Elegidos/as } \\
\hline & \multicolumn{2}{|c|}{ Hombres } & \multicolumn{2}{|c|}{ Mujeres } & \multicolumn{2}{|c|}{ Total } & \multicolumn{2}{|c|}{ Hombres } & \multicolumn{2}{|c|}{ Mujeres } & \multicolumn{2}{|c|}{ Total } \\
\hline & $\mathrm{N}$ & $\%$ & $\mathrm{~N}$ & $\%$ & $\mathrm{~N}$ & $\%$ & $\mathrm{~N}$ & $\%$ & $\mathrm{~N}$ & $\%$ & $\mathrm{~N}$ & $\%$ \\
\hline Gran Canaria & 2.026 & 73,2 & 742 & 26,8 & 2.768 & 100,0 & 297 & 84,1 & 56 & 15,9 & 353 & 100,0 \\
\hline Lanzarote & 563 & 74,9 & 189 & 25,1 & 752 & 100,0 & 79 & 84,9 & 14 & 15,1 & 93 & 100,0 \\
\hline Fuerteventura & 324 & 76,4 & 100 & 23,6 & 424 & 100,0 & 65 & 87,8 & 9 & 12,2 & 74 & 100,0 \\
\hline Provincia de Las & 2.913 & 73,9 & 1.031 & 26,1 & 3.944 & 100,0 & 441 & 84,8 & 79 & 15,2 & 520 & 100,0 \\
\hline Tenerife & 2.120 & 73,5 & 763 & 26,5 & 2.883 & 100,0 & 397 & 81,9 & 88 & 18,1 & 485 & 100,0 \\
\hline La Palma & 596 & 74,3 & 206 & 25,7 & 802 & 100,0 & 140 & 81,4 & 32 & 18,6 & 172 & 100,0 \\
\hline La Gomera & 204 & 78,5 & 56 & 21,5 & 260 & 100,0 & 54 & 84,4 & 10 & 15,6 & 64 & 100,0 \\
\hline El Hierro & 103 & 73,6 & 37 & 26,4 & 140 & 100,0 & 19 & 86,4 & 3 & 13,6 & 22 & 100,0 \\
\hline Provincia de Santa Cruz & 3.023 & 74,0 & 1.062 & 26,0 & 4.085 & 100,0 & 610 & 82,1 & 133 & 17,9 & 743 & 100,0 \\
\hline Canarias & 5.936 & 73,9 & 2.093 & 26,1 & 8.029 & 100,0 & 1.051 & 83,2 & 212 & 16,8 & 1.263 & 100,0 \\
\hline \multirow{2}{*}{$\begin{array}{l}\text { Elecciones a cabildos } \\
\text { insulares }\end{array}$} & \multicolumn{2}{|c|}{ Hombres } & \multicolumn{2}{|c|}{ Mujeres } & \multicolumn{2}{|c|}{ Total } & \multicolumn{2}{|c|}{ Hombres } & \multicolumn{2}{|c|}{ Mujeres } & \multicolumn{2}{|c|}{ Total } \\
\hline & $\mathrm{N}$ & $\%$ & $\mathrm{~N}$ & $\%$ & $\mathrm{~N}$ & 70 & $\mathrm{~N}$ & $\%$ & $\mathrm{~N}$ & $\%$ & $\mathrm{~N}$ & $\%$ \\
\hline Gran Canaria & 213 & 74,0 & 75 & 2 & 288 & 10 & 26 & 89,7 & 3 & 10,3 & 29 & 100,0 \\
\hline Lanzarote & 145 & 75,5 & 47 & 24,5 & 192 & 100,0 & 18 & 85,7 & 3 & 14,3 & 21 & 100,0 \\
\hline Fuerteventura & 87 & 72,5 & 33 & 27,5 & 120 & 100,0 & 16 & 94,1 & 1 & 5,9 & 17 & 100,0 \\
\hline Provincia de Las Palmas & 445 & 74,2 & 155 & 25,8 & 600 & 100,0 & 60 & 89,6 & 7 & 10,4 & 67 & 100,0 \\
\hline Tenerife & 222 & 67,3 & 108 & 32,7 & 330 & 100,0 & 23 & 85,2 & 4 & 14,8 & 27 & 100,0 \\
\hline La Palma & 90 & 75,0 & 30 & 25,0 & 120 & 100,0 & 19 & 90,5 & 2 & 9,5 & 21 & 100,0 \\
\hline La Gomera & 51 & 79,7 & 13 & 20,3 & 64 & 100,0 & 10 & 76,9 & 3 & 23,1 & 13 & 100,0 \\
\hline El Hierro & 61 & 72,6 & 23 & 27,4 & 84 & 100,0 & 9 & 81,8 & 2 & 18,2 & 11 & 100,0 \\
\hline Provincia de Santa Cruz & 424 & 70,9 & 174 & 29,1 & 598 & 100,0 & 61 & 84,7 & 11 & 15,3 & 72 & 100,0 \\
\hline Canarias & 869 & 72,5 & 329 & 27,5 & 1.198 & 100,0 & 121 & 87,1 & 18 & 12,9 & 139 & 100,0 \\
\hline
\end{tabular}


Tabla 7. Candidatos/as y elegidos/as por instituciones y circunscripciones. Canarias, 1995 (continuación).

\begin{tabular}{|c|c|c|c|c|c|c|c|c|c|c|c|c|}
\hline \multirow{3}{*}{$\begin{array}{l}\text { Elecciones } \\
\text { autonómicas }\end{array}$} & \multicolumn{6}{|c|}{ Candidatos/as } & \multicolumn{6}{|c|}{ Elegidos/as } \\
\hline & \multicolumn{2}{|c|}{ Hombres } & \multicolumn{2}{|c|}{ Mujeres } & \multicolumn{2}{|c|}{ Total } & \multicolumn{2}{|c|}{ Hombres } & \multicolumn{2}{|c|}{ Mujeres } & \multicolumn{2}{|c|}{ Total } \\
\hline & $\mathrm{N}$ & $\%$ & $\mathrm{~N}$ & $\%$ & $\mathrm{~N}$ & $\%$ & $\mathrm{~N}$ & $\%$ & $\mathrm{~N}$ & $\%$ & $\mathrm{~N}$ & $\%$ \\
\hline Gran Canaria & 130 & 72,2 & 50 & 27,8 & 180 & 100,0 & 12 & 80,0 & 3 & 20,0 & 15 & 100,0 \\
\hline Lanzarote & 57 & 74,0 & 20 & 26,0 & 77 & 100,0 & 7 & 87,5 & 1 & 12,5 & 8 & 100,0 \\
\hline Fuerteventura & 47 & 67,1 & 23 & 32,9 & 70 & 100,0 & 6 & 85,7 & 1 & 14,3 & 7 & 100,0 \\
\hline Provincia de Las Palmas & 234 & 71,6 & 93 & 28,4 & 327 & 100,0 & 25 & 83,3 & 5 & 16,7 & 30 & 100,0 \\
\hline Tenerife & 121 & 66,9 & 60 & 33,1 & 181 & 100,0 & 13 & 86,7 & 2 & 13,3 & 15 & 100,0 \\
\hline La Palma & 38 & 69,1 & 17 & 30,9 & 55 & 100,0 & 8 & 100,0 & 0 & 0,0 & 8 & 100,0 \\
\hline La Gomera & 24 & 68,6 & 11 & 31,4 & 35 & 100,0 & 4 & 100,0 & 0 & 0,0 & 4 & 100,0 \\
\hline El Hierro & 28 & 77,8 & 8 & 22,2 & 36 & 100,0 & 3 & 100,0 & 0 & 0,0 & 3 & 100,0 \\
\hline Provincia de Santa Cruz & 211 & 68,7 & 96 & 31,3 & 307 & 100,0 & 28 & 93,3 & 2 & 6,7 & 30 & 100,0 \\
\hline Canarias & 445 & 70,2 & 189 & 29,8 & 634 & 100,0 & 53 & 88,3 & 7 & 11,7 & 60 & 100,0 \\
\hline
\end{tabular}

Fuente: elaboración propia.

Tabla 8. Elecciones municipales. Datos básicos.

\begin{tabular}{lrr} 
& Total & $\%$ \\
\hline Total de candidaturas que comparecen & 417 & \\
Total de candidatos que comparecen & 8.029 & \\
Total de candidatos varones & 5.936 & 73,93 \\
Total de candidatos mujeres & 2.093 & 26,07 \\
\hline & & 100,00 \\
\hline
\end{tabular}

Tabla 9. Posición de $1 @_{s}$ candidat@s en las listas electorales agrupadas según la magnitud poblacional de los municipios. Canarias, 1995.

\begin{tabular}{|c|c|c|c|c|c|c|c|c|c|c|c|c|c|c|c|}
\hline \multirow[b]{2}{*}{ Habitantes } & \multicolumn{5}{|c|}{ Grupo $1\left(1^{0}-3^{\circ}\right)$} & \multicolumn{5}{|c|}{ Grupo $2\left(4^{\circ}-10^{\circ}\right)$} & \multicolumn{5}{|c|}{ Grupo $3\left(11^{\circ}-20^{\circ}\right)$} \\
\hline & $\mathrm{M}$ & $\mathrm{H}$ & Total & $\% \mathrm{M}$ & $\% \mathrm{H}$ & $\mathrm{M}$ & $\mathrm{H}$ & Total & $\% \mathrm{M}$ & $\% \mathrm{H}$ & M & $\mathrm{H}$ & Total & $\% \mathrm{M}$ & $\% \mathrm{H}$ \\
\hline $1-10.000$ & 101 & 583 & 684 & 1,3 & 7,3 & 381 & 1.179 & 1.560 &, 7 & 14,7 & 115 & 341 & 462 & 1,4 & 4,3 \\
\hline $10.001-30.000$ & 65 & 316 & 381 & 0,8 & 3,9 & 218 & 676 & 894 & 2,7 & 8,4 & 310 & 702 & 1.012 & 3,9 & 8,7 \\
\hline 30.001-100. & 26 & 115 & 141 & 0,3 & 1,4 & 86 & 242 & 329 & 1,1 & 3,0 & 156 & 314 & 470 & 1,9 & 3,9 \\
\hline $100.001+$ & 17 & 59 & 76 & 0,2 & 0,7 & 52 & 125 & 177 &, 6 & 1,6 & 84 & 62 & 246 & 1,0 & 2,0 \\
\hline \multirow[t]{2}{*}{ Sumas } & 209 & 1.073 & 1.282 & 2,6 & 13,4 & 737 & 2.222 & 2.960 & 9,2 & 27,7 & 665 & 1.525 & 2.190 & 8,3 & 19,0 \\
\hline & \multicolumn{5}{|c|}{ Grupo $4\left(21^{\circ}+\right)$} & \multicolumn{5}{|c|}{ Suplentes $1\left(1^{0}-3^{\circ}\right)$} & \multicolumn{5}{|c|}{ Suplentes $2\left(4^{0}+\right)$} \\
\hline Habitantes & $\mathrm{M}$ & $\mathrm{H}$ & Total & $\% \mathrm{M}$ & $\% \mathrm{H}$ & $\mathrm{M}$ & $\mathrm{H}$ & Total & $\% \mathrm{M}$ & $\% \mathrm{H}$ & $\mathrm{M}$ & $\mathrm{H}$ & Total & $\% \mathrm{M}$ & $\% \mathrm{H}$ \\
\hline $1-10.000$ & 0 & 0 & 0 & 0,0 & 0,0 & 198 & 5 & 704 & 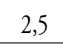 & 6 , & 0 & 0 & 0 & 0,0 & 0,0 \\
\hline $10.001-30.000$ & 10 & 30 & 40 & 0,1 & 0,4 & 110 & 243 & 353 & 1,4 & 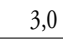 & 2 & 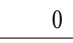 & 2 & 0,0 & 0,0 \\
\hline $30.001-100.000$ & 58 & 34 & 92 & 0,7 & 0,4 & 36 & 104 & 140 & 0,4 & 1, & 0 & 0 & 0 & 0,0 & 0,0 \\
\hline $100.001+$ & 67 & 124 & 191 & 0,8 & 1,5 & 34 & 42 & 76 & 0,4 & 0, & 0 & 0 & 0 & 0,0 & 0,0 \\
\hline Sumas & 135 & 188 & 323 & 1,7 & 2,3 & 378 & 895 & 1.273 & 4,7 & 11,2 & 2 & 0 & 2 & 0,0 & 0,0 \\
\hline
\end{tabular}

Fuente: elaboración propia. 


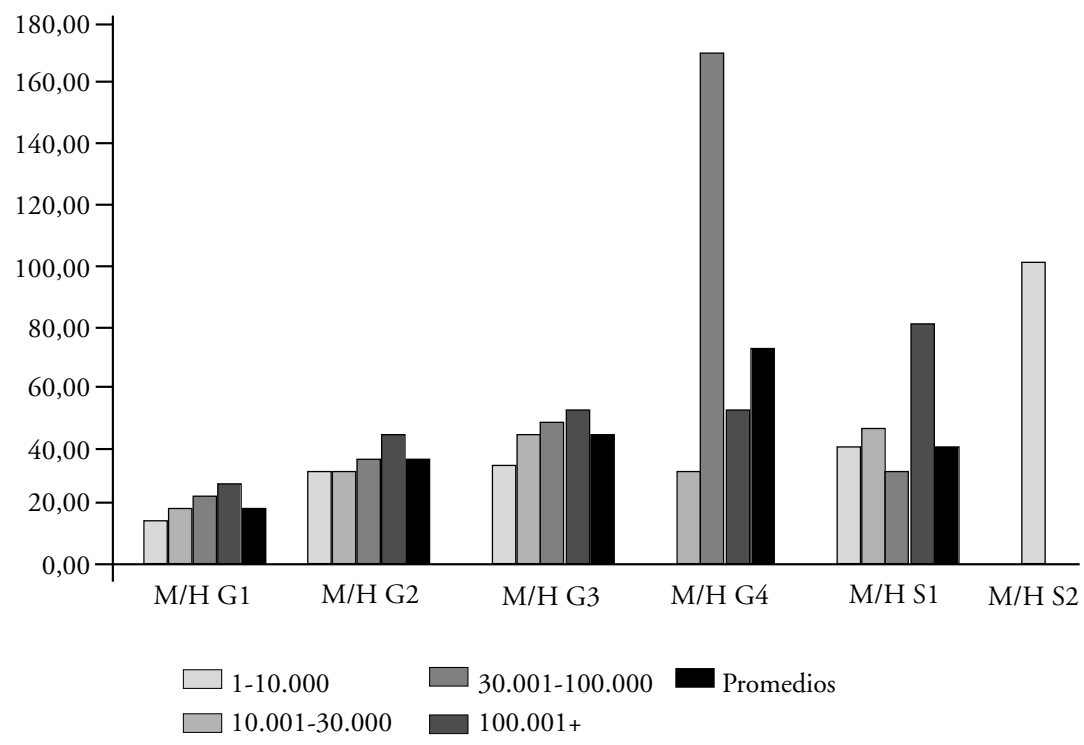

Gráfico 1. Tanto por ciento de mujeres sobre número de hombres, por grupos y clases de municipios.

Fuente: elaboración propia.

- Siguiendo esta tendencia, observamos que la proporción de mujeres en cada grupo es bastante mayor en los municipios de mayor tamaño.

Si atendemos a la proporción de candidatos y candidatas en las distintas posiciones de las listas electorales ordenadas en grupos y comparamos la situación en los municipios, ordenados en grupos de magnitud poblacional, obtenemos los resultados reflejados en el gráfico 1:

1. Los hombres dominan en todos los grupos y todos los municipios salvo escasas excepciones.

2. La proporción de mujeres aumenta según se desciende en la escala de puestos en lista, independientemente de la magnitud poblacional del municipio en cuestión.

3. Hombres y mujeres muestran modos característicos de distribución en las listas electorales, que, a su vez, varían en función de la población del municipio (al variar con ella el número de puestos a cubrir). Mientras los hombres se concentran en los grupos segundo y tercero, las mujeres lo hacen en el tercero y cuarto donde la población del municipio lo permite (a partir de 30.001 habitantes). En los municipios menores, se concentran en el tercer grupo.

4. Un resultado hasta cierto punto inesperado es el hecho de que el tamaño poblacional del municipio afecta positivamente a la proporción de mujeres en las listas. En efecto, los municipios de más población muestran mayor proporción de mujeres en listas que los municipios menores en todos los 
grupos de puestos en lista. La presunción de que la cercanía a la institución facilita el acceso de la mujer a ella no se cumple en este caso, ya que el ambiente urbano, a pesar de suponer la presencia de mayores instancias intermediarias entre la ciudadanía y las instituciones, actúa como liberador de las barreras culturales y presiones sociales que dificultan la participación femenina en los entornos rurales.

Por lo tanto, la magnitud poblacional no parece ser una variable independiente a la hora de decidir la posición que una mujer dada ocupará en una lista electoral, aunque sí influye en el número de mujeres que entrarán en la lista.

De lo indicado se deriva que la ya de por sí escasa proporción de mujeres entre los candidatos se verá aún más reducida entre los elegidos, dado su mal posicionamiento en las listas electorales. Efectivamente, de los 1.263 concejales electos, sólo 212 (16,78\%) son mujeres, viéndose su participación reducida en casi diez puntos porcentuales con relación a su condición de candidata $(26,07 \%)$, como se refleja en la tabla 10 .

Comparando los datos correspondientes a las dos provincias canarias, apenas se observan diferencias entre ellas, siendo éstas de uno o dos puntos porcentuales, a lo sumo (tabla 11).

Tabla 10. Concejales electos en Canarias, 1995.

\begin{tabular}{lrr}
\hline & Total & $\%$ \\
\hline Total de elegid@s & 1.263 & \\
Total de elegidos & 1.051 & 83,21 \\
Total de elegidas & 212 & 16,78 \\
\hline
\end{tabular}

Fuente: elaboración propia.

Tabla 11. Elecciones municipales. Canarias, 1995.

\begin{tabular}{|c|c|c|c|c|c|c|c|c|c|c|c|c|}
\hline & \multicolumn{6}{|c|}{ Canditatos/as } & \multicolumn{6}{|c|}{ Elegidos/as } \\
\hline & \multicolumn{2}{|c|}{ Hombres } & \multicolumn{2}{|c|}{ Mujeres } & \multicolumn{2}{|c|}{ Total } & \multicolumn{2}{|c|}{ Hombres } & \multicolumn{2}{|c|}{ Mujeres } & \multicolumn{2}{|c|}{ Total } \\
\hline & $\mathrm{N}$ & $\%$ & $\mathrm{~N}$ & $\%$ & $\mathrm{~N}$ & $\%$ & $\mathrm{~N}$ & $\%$ & $\mathrm{~N}$ & $\%$ & $\mathrm{~N}$ & $\%$ \\
\hline Gran Canaria & 2.026 & 73,2 & 742 & 26,8 & 2.768 & 100,0 & 297 & 84,1 & 56 & 15,9 & 353 & 100,0 \\
\hline Lanzarote & 563 & 74,9 & 189 & 25,1 & 752 & 100,0 & 79 & 84,9 & 14 & 15,1 & 93 & 100,0 \\
\hline Fuerteventura & 324 & 76,4 & 100 & 23,6 & 424 & 100,0 & 65 & 87,8 & 9 & 12,2 & 74 & 100,0 \\
\hline Provincia de Las Palmas & 2.913 & 73,9 & 1.031 & 26,1 & 3.944 & 100,0 & 441 & 84,8 & 79 & 15,2 & 520 & 100,0 \\
\hline Tenerife & 2.120 & 73,5 & 763 & 26,5 & 2.883 & 100,0 & 397 & 81,9 & 88 & 18,1 & 485 & 100,0 \\
\hline La Palma & 596 & 74,3 & 206 & 25,7 & 802 & 100,0 & 140 & 81,4 & 32 & 18,6 & 172 & 100,0 \\
\hline La Gomera & 204 & 78,5 & 56 & 21,5 & 260 & 100,0 & 54 & 84,4 & 10 & 15,6 & 64 & 100,0 \\
\hline El Hierro & 103 & 73,6 & 37 & 26,4 & 140 & 100,0 & 19 & 86,4 & 3 & 13,6 & 22 & 100,0 \\
\hline Provincia de Santa Cruz & 3.023 & 74,0 & 1.062 & 26,0 & 4.085 & 100,0 & 610 & 82,1 & 133 & 17,9 & 743 & 100,0 \\
\hline Canarias & 5.936 & 73,9 & 2.093 & 26,1 & 8.028 & 100,0 & 1.051 & 82,2 & 212 & 16,8 & 1.262 & 100,0 \\
\hline
\end{tabular}

Fuente: elaboración propia. 
En la provincia de Las Palmas de Gran Canaria se presentaron 197 candidaturas, comparenciendo 3.944 candidatos y candidatas, de los cuales 1.031 $(26,1 \%)$ eran mujeres, frente al $73,9 \%$ (2.913) de hombres. Con respecto a la posición de las mujeres en las listas, ésta corresponde con la tendencia regional, al igual que la proporción de mujeres según magnitud poblacional de los municipios. Apreciamos una similar presencia femenina en función de la población del municipio que la observada a nivel regional.

En la provincia de Santa Cruz de Tenerife se presentan 220 candidaturas, compareciendo 4.085 candidatos y candidatas. El mayor número de candidaturas y candidatos se debe a que el territorio de esta provincia está dividido en cuatro islas, estando la isla de Tenerife, por ejemplo, fragmentada en más de treinta municipios de escasa población.

En Santa Cruz encontramos 1.062 candidatas, un 25,9\% del total, frente a un $74 \%$ de hombres (3.023). Como vemos, proporción prácticamente igual a la de Las Palmas. En cuanto a la posición en las listas, la distribución de las mujeres es similar a la de los hombres, con una diferencia: mientras los hombres se concentran entre los puestos del $4^{\circ}$ al $10^{\circ}$ (G. 2), las mujeres se reparten por igual entre los puestos del $4^{\circ}$ al $10^{\circ}$ y del $11^{\circ}$ al $20^{\circ}$ (G. 3). Así, los hombres triplican a las mujeres en el G. 2 y las quintuplican en el G. 1. En el G. 4, en cambio, «sólo» son el doble. La peculiar estructura territorial de Santa Cruz lleva a algunas desviaciones en relación con la influencia de la población sobre la presencia femenina, pero el promedio es similar al regional.

En la provincia de Las Palmas, entre los 520 concejales electos, las mujeres suponen, con 79 elegidas, el 15,2\%, mientras que los hombres alcanzan el $84,4 \%$ con 441 ediles. Otro tanto sucede en Santa Cruz, donde los porcentajes son similares, con una presencia femenina algo superior.

\subsubsection{Elecciones a cabildos insulares de la Comunidad Canaria}

El número de candidaturas presentadas asciende a 49, con 1.198 candidatos/as a consejero insular, de los cuales 329 , el $27,46 \%$, son mujeres, mientras los hombres, con 869 candidatos, suponen el 72,53\% del total (tabla 12 y gráfico 2).

La distribución de las mujeres en las listas es diferente a la de los hombres (tabla 13). Mientras éstos se concentran por igual en los grupos segundo y tercero, el porcentaje de mujeres en el segundo grupo $(23,1 \%)$ es claramente inferior al del tercer grupo (37,4\% de las candidatas). Se da una relación de proporcionalidad inversa entre la posición en la lista y el número de mujeres.

En el caso de los cabildos, se confirma que la distancia entre la institución y la ciudadanía dificulta el acceso de la mujer a las candidaturas. Ahora bien, atendiendo a los resultados obtenidos del análisis de las elecciones municipales según población del municipio, comentados en el apartado anterior, se aprecia que este mecanismo de exclusión opera a través de la lejanía intrínseca de la institución y no, o por lo menos no perceptiblemente, a través de la magnitud poblacional de la circunscripción. Tanto en el caso de los cabildos como de los ayuntamientos, la legislación política prevé mecanismos correctores del tamaño poblacional de las circunscripciones a través del número de car- 
Tabla 12. Elecciones a los cabildos insulares. Canarias, 1995.

\begin{tabular}{|c|c|c|c|c|c|c|c|c|c|c|c|c|}
\hline & \multicolumn{6}{|c|}{ Canditatos/as } & \multicolumn{6}{|c|}{ Elegidos/as } \\
\hline & \multicolumn{2}{|c|}{ Hombres } & \multicolumn{2}{|c|}{ Mujeres } & \multicolumn{2}{|c|}{ Total } & \multicolumn{2}{|c|}{ Hombres } & \multicolumn{2}{|c|}{ Mujeres } & \multicolumn{2}{|c|}{ Total } \\
\hline & $\mathrm{N}$ & $\%$ & $\mathrm{~N}$ & $\%$ & $\mathrm{~N}$ & $\%$ & $\mathrm{~N}$ & $\%$ & $\mathrm{~N}$ & $\%$ & $\mathrm{~N}$ & $\%$ \\
\hline Gran Canaria & 213 & 74,0 & 75 & 26,0 & 288 & 100,0 & 26 & 89,7 & 3 & 10,3 & 29 & 100,0 \\
\hline Lanzarote & 145 & 75,5 & 47 & 24,5 & 192 & 100,0 & 18 & 85,7 & 3 & 14,3 & 21 & 100,0 \\
\hline Fuerteventura & 87 & 72,5 & 33 & & 120 & 100,0 & 16 & 94,1 & 1 & 5,9 & 17 & 100,0 \\
\hline Provincia de La & 445 & 74,2 & 155 & 25,8 & 600 & 100,0 & 60 & 89,6 & 7 & 10,4 & 67 & 100,0 \\
\hline Tenerife & 222 & 67,3 & 108 & 32,7 & 330 & 100,0 & 23 & 85,2 & 4 & 14,8 & 27 & 100,0 \\
\hline La Palma & 90 & 75,0 & 30 & 25,0 & 120 & 100,0 & 19 & 90,5 & 2 & 9,5 & 21 & 100,0 \\
\hline La Gomera & 51 & 79,7 & 13 & 20,3 & 64 & 100,0 & 10 & 76,9 & 3 & 23,1 & 13 & 100,0 \\
\hline El Hierro & 61 & 72,6 & 23 & 27,4 & 84 & 100,0 & 9 & 81,8 & 2 & 18,2 & 11 & 100,0 \\
\hline Provincia de Santa Cruz & 424 & 70,9 & 174 & 29,1 & 598 & 100,0 & 61 & 84,7 & 11 & 15,3 & 72 & 100,0 \\
\hline Canarias & 869 & 72,5 & 329 & 27,5 & 1.198 & 100,0 & 121 & 87,1 & 18 & 12,9 & 139 & 100,0 \\
\hline
\end{tabular}

Fuente: elaboración propia.

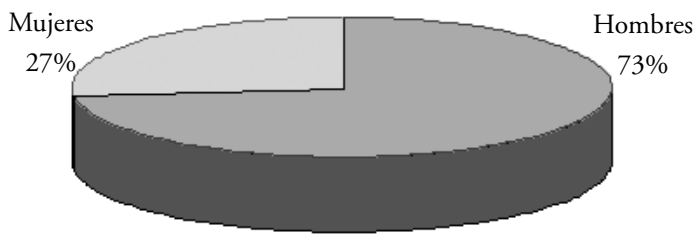

Gráfico 2. Candidatos a cabildos. Canarias, 1995.

Fuente: elaboración propia.

Tabla 13. Posición de $1 @_{s}$ candidat@s en las listas electorales según grupos.

\begin{tabular}{|c|c|c|c|c|c|c|c|}
\hline \multicolumn{2}{|c|}{ Cabildos } & \multicolumn{2}{|c|}{ Mujeres } & \multicolumn{2}{|c|}{ Hombres } & \multicolumn{2}{|c|}{ Total } \\
\hline Grupos & Puestos & Total & $\%$ & Total & $\%$ & Total & $\%$ \\
\hline Grupo 1 & $1-3$ & 19 & 1,58 & 130 & 10,85 & 149 & 12,44 \\
\hline Grupo 2 & $4-10$ & 76 & 6,34 & 264 & 22,03 & 340 & 28,38 \\
\hline Grupo 3 & $11-20$ & 123 & 10,26 & 266 & 22,30 & 389 & 32,47 \\
\hline Grupo 4 & 21 y más & 58 & 4,84 & 115 & 9,59 & 173 & 14,44 \\
\hline Suplentes 1 & $1-3$ & 53 & 4,42 & 94 & 7,84 & 147 & 12,27 \\
\hline Suplentes 2 & 4 y más & 0 & 0 & 0 & 0 & 0 & 0 \\
\hline Totales & & 329 & 27,46 & 869 & 72,54 & 1.198 & 100 \\
\hline
\end{tabular}

Fuente: elaboración propia. 
gos a cubrir: a menor población, menor número de cargos y, por lo tanto, mayor competencia para aspirar a cubrirlos.

Si examinamos la proporción mujeres/hombres en cada grupo, se observa una situación similar: mientras la proporción es de 0,13 en el primer grupo, en el cuarto alcanza el valor de 0,66 (6,6 mujeres por cada 10 hombres) (tabla 14).

De los 1.198 candidatos fueron elegidos 139 , 18 de los cuales (12,94\%) fueron mujeres y $121(87,05 \%)$, hombres (gráfico 3). Si comparamos los porcentajes de mujeres elegidas en las elecciones municipales $(16,8 \%)$ y las insulares $(12,9 \%)$ medimos un porcentaje inferior en cuatro puntos en el caso de las últimas, lo que refuerza la validez de la lejanía institucional como factor explicativo de la proporción de mujeres en listas electorales en Canarias. Sin embargo, es preciso hacer notar que, como se desprende de la tabla 12, se producen notables diferencias de una isla a otra y entre provincias; mientras en Las Palmas resulta elegido un $10 \%$ de mujeres, en Santa Cruz se alcanza el $15,3 \%$, es decir, un $50 \%$ más.

Tabla 14. Posición de $1 @_{s}$ candidat@s en las listas electorales según grupos.

\begin{tabular}{|c|c|c|c|c|c|c|c|c|c|c|c|c|c|c|c|}
\hline \multirow{2}{*}{$\begin{array}{l}\text { Cabildos } \\
\text { Provincia } \\
\end{array}$} & \multicolumn{5}{|c|}{ Grupo $1\left(1^{10}-3^{\circ}\right)$} & \multicolumn{5}{|c|}{ Grupo $2\left(4^{\circ}-10^{\circ}\right)$} & \multicolumn{5}{|c|}{ Grupo $3\left(11^{\circ}-20^{\circ}\right)$} \\
\hline & M & $\mathrm{H}$ & Total & $\% \mathrm{M}$ & $\% \mathrm{H}$ & M & $\mathrm{H}$ & Total & $\% \mathrm{M}$ & $\% \mathrm{H}$ & $\mathrm{M}$ & $\mathrm{H}$ & Total & $\% \mathrm{M}$ & $\% \mathrm{H}$ \\
\hline Las Palmas & 9 & 70 & 79 & 0,75 & 5,84 & 43 & 138 & 181 & 3,58 & 11,51 & 58 & 119 & 177 & 4,84 & 49,93 \\
\hline Santa Cruz & 10 & 60 & 70 & 0,83 & 5,00 & 33 & 126 & 159 & 2,75 & 10,51 & 65 & 147 & 212 & 5,42 & 212,27 \\
\hline \multirow[t]{2}{*}{ Sumas } & 19 & 130 & 149 & 1,58 & 10,84 & 76 & 264 & 340 & 6,33 & 22,02 & 123 & 266 & 389 & 10,26 & 622,20 \\
\hline & \multicolumn{5}{|c|}{ Grupo $4\left(21^{10}+\right)$} & \multicolumn{5}{|c|}{ Suplente $\mathrm{G} 1\left(1^{\mathrm{o}} 3^{\circ}\right)$} & \multicolumn{5}{|c|}{ Suplente G2 $\left(4^{0}+\right)$} \\
\hline Provincia & $\mathrm{M}$ & $\mathrm{H}$ & Total & $\% \mathrm{M}$ & $\% \mathrm{H}$ & $\mathrm{M}$ & $\mathrm{H}$ & Total & $\% \mathrm{M}$ & $\% \mathrm{H}$ & M & $\mathrm{H}$ & Total & $\% \mathrm{M}$ & $\% \mathrm{H}$ \\
\hline Las Palmas & 33 & 50 & 83 & 2,75 & 4,17 & 31 & 47 & 78 & 2,58 & 3,9 & 0 & 0 & 0 & 0 & 0 \\
\hline Santa Cruz & 25 & 65 & 90 & 2,08 & 5,42 & 22 & 47 & 69 & 1,83 & 3,92 & 0 & 0 & 0 & 0 & 0 \\
\hline Sumas & 58 & 115 & 173 & 4,80 & 9,59 & 53 & 94 & 147 & 4,41 & 7,84 & 0 & 0 & 0 & 0 & 0 \\
\hline
\end{tabular}

Fuente: elaboración propia.

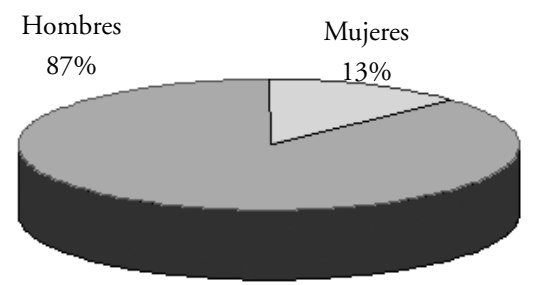

Gáfico 3. Elegidos a cabildos. Canarias, 1995.

Fuente: elaboración propia. 
Tabla 15. Presencia femenina en cabildos insulares en función del número de consejeros, población insular y relaciones de mayoría.

\begin{tabular}{cccll}
\hline Consejeras & Total de consejeros/as & \% mujeres & Isla & Partidos dominantes \\
\hline 3 & 13 & 23,7 & La Gomera & PSC-PSOE \\
\hline 2 & 11 & 18,0 & El Hierro & AHI \\
\hline 3 & 21 & 14,3 & Lanzarote & PIL \\
\hline 4 & 27 & 14,2 & Tenerife & CC, PP \\
\hline 3 & 29 & 10,3 & Gran Canaria & PP, CC \\
\hline 2 & 21 & 9,52 & La Palma & API \\
\hline 1 & 17 & 5,0 & Fuerteventura & AM, PP, PSC-PSOE \\
\hline 18 & 139 & 12,9 & Canarias & - \\
\hline
\end{tabular}

mayoría absoluta

Fuente: elaboración propia.

Dada la distribución de las mujeres en las listas, nos encontramos con que éstas sólo encuentran una representación aproximadamente adecuada como elegidas entre los partidos mejor situados, especialmente en los casos de mayorías claras y partidos que, por cualesquiera motivos, hayan decidido contar con una fuerte presencia femenina.

Como nos indica la tabla 15, nos encontramos con que las mujeres en ningún caso superan el 23,7\% alcanzado en La Gomera. Por lo demás, no se aprecia una relación entre la población de la isla o el número de consejeros a elegir con el porcentaje de mujeres elegidas, ya que la posición de las mujeres en la lista se adapta al número de consejeros en liza en función de los llamados «puestos de salida». Éste concepto nos marca la divisoria entre candidatos que realmente optan a ganar y puestos «de relleno». Éstos últimos serán, en cualquier caso, los superiores a la mitad de los cargos a elegir, ya que necesitarían una mayoría abrumadora para ocupar un cargo público. Las mujeres se concentran siempre en los puestos que no son de salida. El porcentaje de mujeres en puestos de salida es prácticamente uniforme en Canarias. El porcentaje de mujeres efectivamente elegidas dependerá también de la distribución del voto entre los partidos y de la política de integración de mujeres de cada partido. Este último factor tiene en la convocatoria de 1995 un factor marcadamente local, produciéndose diferencias notables no sólo entre partidos, sino también en un mismo partido.

\subsubsection{Elecciones al Parlamento de Canarias}

El número de candidaturas fue de 50, con 634 candidatos, 445 de los cuales fueron varones $(70,18 \%)$ y 189 mujeres $(29,8 \%)$ (gráfico 4$)$. Las mujeres elegidas fueron $7(11,7 \%)$, frente a 53 hombres $(88,3 \%)$ (gráfico 5$)$. 


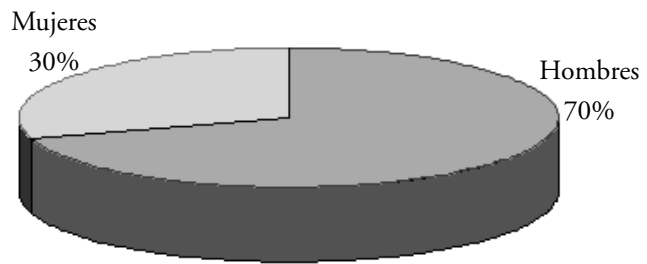

Gáfico 4. Candidatos al Parlamento de Canarias, 1995.

Fuente: elaboración propia.

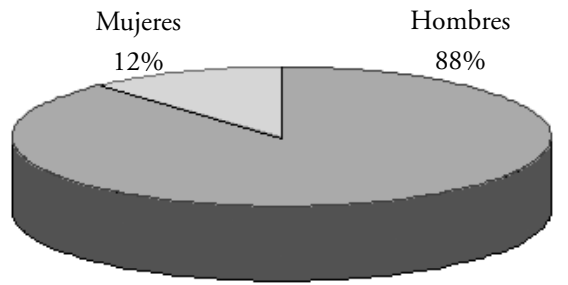

Gáfico 5. Elegidos al Parlamento de Canarias, 1995.

Fuente: elaboración propia.

En primer lugar, llama la atención el hecho de que la proporción de mujeres entre los candidatos es mayor que en cualquier otra institución, mientras la proporción de elegidas es la menor de todas.

Atendiendo a la distribución de las mujeres en las listas electorales, encontramos que las circunscripciones son tan diferentes en cuanto al número de puestos a cubrir que resulta impracticable extraer consecuencias de los posicionamientos en lista agregados. Los datos insulares, en cambio, no dejan lugar a dudas: donde el número de puestos a cubrir lo permite, las mujeres se concentran en los grupos 2 y 3 y los hombres en los dos primeros. En las islas menores simplemente se traslada este esquema jerárquico hacia arriba en la lista. Así, en puestos de salida, los hombres como mínimo cuadruplican a las mujeres, mientras éstas acortan distancias o incluso superan numéricamente a los hombres en los últimos puestos de las listas.

Es, pues, en el Parlamento de Canarias donde, pese a una mayor presencia de la mujer en las listas, opera más consecuentemente el sistema de exclusión a través de los puestos de salida (tablas 16 y 17).

En resumen: los factores que actuaban en el caso de las elecciones a cabildos los encontramos reforzados en el caso del Parlamento regional, ya que el escaso número de puestos a cubrir y su reparto fragmentado entre las islas produce importantes distorsiones del principio de proporcionalidad en algunas 
Tabla 16. Elecciones autonómicas. Canarias, 1995.

\begin{tabular}{|c|c|c|c|c|c|c|c|c|c|c|c|c|}
\hline & \multicolumn{6}{|c|}{ Canditatos/as } & \multicolumn{6}{|c|}{ Elegidos/as } \\
\hline & \multicolumn{2}{|c|}{ Hombres } & \multicolumn{2}{|c|}{ Mujeres } & \multicolumn{2}{|c|}{ Total } & \multicolumn{2}{|c|}{ Hombres } & \multicolumn{2}{|c|}{ Mujeres } & \multicolumn{2}{|c|}{ Total } \\
\hline & $\mathrm{N}$ & $\%$ & $\mathrm{~N}$ & $\%$ & $\mathrm{~N}$ & $\%$ & $\mathrm{~N}$ & $\%$ & $\mathrm{~N}$ & $\%$ & $\mathrm{~N}$ & $\%$ \\
\hline Gran Canaria & 130 & 72,2 & 50 & 27,8 & 180 & 100,0 & 12 & 80,0 & 3 & 20,0 & 15 & 100,0 \\
\hline Lanzarote & 57 & 74,0 & 20 & 26,0 & 77 & 100,0 & 7 & 87,5 & 1 & 12,5 & 8 & 100,0 \\
\hline Fuerteventura & 47 & 67,1 & 23 & 32,9 & 70 & 100,0 & 6 & 85,7 & 1 & 14,3 & 7 & 100,0 \\
\hline Provincia de Las Palmas & 234 & 71,6 & 93 & 28,4 & 327 & 100,0 & 25 & 83,3 & 5 & 16,7 & 30 & 100,0 \\
\hline Tenerife & 121 & 66,9 & 60 & 33,1 & 181 & 100,0 & 13 & 86,7 & 2 & 13,3 & 15 & 100,0 \\
\hline La Palma & 38 & 69,1 & 17 & 30,9 & 55 & 100,0 & 8 & 100,0 & 0 & 0,0 & 8 & 100,0 \\
\hline La Gomera & 24 & 68,6 & 11 & 31,4 & 35 & 100,0 & 4 & 100,0 & 0 & 0,0 & 4 & 100,0 \\
\hline El Hierro & 28 & 77,8 & 8 & 22,2 & 36 & 100,0 & 3 & 100,0 & 0 & 0,0 & 3 & 100,0 \\
\hline Provincia de Santa Cruz & 211 & 68,7 & 96 & 31,3 & 307 & 100,0 & 28 & 93,3 & 2 & 6,7 & 30 & 100,0 \\
\hline Canarias & 445 & 70,2 & 199 & 28,8 & 634 & 100,0 & 53 & 88,3 & 7 & 11,7 & 60 & 100,0 \\
\hline
\end{tabular}

Fuente: elaboración propia.

Tabla 17. Composición territorial y puestos de salida para el Parlamento de Canarias.

\begin{tabular}{|c|c|c|c|c|c|}
\hline \multicolumn{3}{|r|}{60 escaños } & \multirow{2}{*}{\multicolumn{3}{|c|}{ Provincia de Santa Cruz (30) }} \\
\hline \multicolumn{3}{|c|}{ Provincia de Las Palmas (30) } & & & \\
\hline Isla & Escaños & P. de salida & Isla & Escaños & P. de salida \\
\hline Gran Canaria & 15 & 8 & Tenerife & 15 & 8 \\
\hline Lanzarote & 10 & 5 & La Palma & 10 & 5 \\
\hline Fuerteventura & 5 & 3 & La Gomera & 3 & 2 \\
\hline & & & El Hierro & 2 & 1 \\
\hline
\end{tabular}

Requisitos mínimos: obtención del 6\% de los votos válidos regionales, ser la fuerza más votada en una isla o, en su defecto, obtener al menos el $30 \%$ de los votos válidos insulares.

Fuente: Ley Electoral de Canarias.

islas, especialmente en La Gomera y en El Hierro. Así, nos encontramos con que en la provincia de Santa Cruz sólo resultan elegidas 2 mujeres, cuando tiene un $31,3 \%$ de candidatas. El hecho de que los puestos de salida en varias islas se reduzcan, en el mejor de los casos, a los dos primeros relativiza la aparentemente buena presencia de la mujer en puestos relativamente altos de las listas. El listón de los puestos de salida es en esta convocatoria más severo que en cualquier otra.

Hacemos constar que situamos los puestos de salida en el listón de la mayoría absoluta para cada partido, cuando en realidad a la hora de confeccionar las listas los partidos emplean criterios mucho menos optimistas. 


\section{Conclusiones}

La conclusión principal y evidente de esta investigación es que, como no sorprenderá a nadie, la presencia femenina en las elecciones autonómicas y locales de 1995, tanto en calidad de candidatas como, especialmente, de elegidas, es francamente escasa. Se podría interpretar esto como consecuencia de una discriminación sistemática por parte de los partidos políticos, pero sería precipitado hacerlo, ya que el material analizado supone únicamente una proyección de unas relaciones de poder internas de los partidos en combinación con unos resultados electorales y los efectos derivados del particular sistema electoral canario, todo ello enmarcado en el sistema de género de la sociedad canaria. Por ello, nos limitamos a exponer una serie de mecanismos que actúan en este proceso, sin pretender explicar a qué procesos sociales concretos responden. En este sentido, el instrumentario empleado nos ha conducido a resultados satisfactorios.

Encontramos dos mecanismos en relación con la presencia femenina, uno referido a las listas electorales y otro referido a los resultados que se complementan con los hallados por M.A. García de León ${ }^{7}$, que había identificado una doble discriminación: cuantitativa, relativa al escaso número de mujeres en las listas, y cualitativa, referente a su mal posicionamiento en ellas. Nuestro estudio añade un nuevo mecanismo y profundiza en el cualitativo.

\section{Mecanismo de lejanía institucional}

Habíamos supuesto que para la mujer era más fácil acceder a puestos de responsabilidad en instituciones cercanas al ciudadano, ya que son el primer paso en la carrera política y, al mismo tiempo, presentan menores barreras para acceder a ellas. Asimismo, la actividad social de las mujeres, p. ej. en asociaciones vecinales, supone un ayuda para el acceso a la política sobre todo en pequeñas localidades ${ }^{8}$. Los resultados parecen confirmar parcialmente estas suposiciones: cuanto más alejada está la institución de la ciudadanía, mayor es el porcentaje de candidatas y menor el de elegidas. Esto se aplica a prácticamente todas las islas. Sin embargo, se aprecia una irrelevancia cualitativa de la magnitud poblacional: Habíamos supuesto que cuanto menor fuera una circunscripción electoral, mayor sería la cercanía de sus instituciones hacia el ciudadano y, por tanto, mayor sería la presencia femenina. Sin embargo, esta suposición resultó ser incompleta. En las elecciones insulares y regionales no se aprecia ninguna relación entre la población de una circunscripción y la presencia femenina como candidata o elegida. En el caso de las elecciones municipales sí se aprecia una correlación, pero diferente a la asumida inicialmente. Los municipios de mayor entidad tienen los mayores porcentajes de mujeres en todos los grupos de la lista y, por tanto, un mayor porcentaje de elegidas. Así pues, el entor-

7. García de LeÓn, M.A. (1994). Elites discriminadas. Barcelona: Anthropos.

8. Molina Petit, op. cit., p. 34 y s. 
no urbano parece poseer características compensatorias de la mayor distancia institucional hacia el ciudadano.

El mecanismo de la lejanía institucional se añade a los hallados por García de León en el estudio citado.

\section{Mecanismo de puestos de salida}

El sistema electoral canario es bastante complejo: las circunscripciones tienen pesos electorales muy diferentes y hay tres niveles institucionales a analizar. Para poder someterlos todos a un mismo análisis, se nos ofreció el concepto de los puestos de salida. Tras aplicarlo al análisis de los datos, obtuvimos resultados bastante consistentes: recordando que el listón de los puestos de salida varía en función de los cargos a elegir y que el número de cargos varía según el tipo de institución y la población de la circunscripción, el resultado de nuestro análisis es que cuanto más alto está el listón de los puestos de salida, menor es el porcentaje de mujeres entre ellos. Vemos que las mujeres experimentan grandes dificultades para acceder a puestos de salida cuanto mayor es la competencia por éstos.

El mecanismo de los puestos de salida supone una ampliación y una precisión del criterio cualitativo de discriminación identificado por García de León. Este instrumento nos permite valorar el posicionamiento en lista de las mujeres independientemente de la institución a la que se presenten, así como comparar el posicionamiento entre instituciones, ya que neutraliza los factores que impiden una comparación directa. A nuestro juicio, este instrumento es de gran utilidad práctica, y presenta un gran potencial de desarrollo. Por ejemplo, hemos asumido como listón de los puestos de salida la obtención de una mayoría absoluta. Se podría mejorar la precisión del instrumento calculando el listón de salida para cada partido en función de sus anteriores resultados.

Como posible explicación de estos datos, nos atenemos a lo anteriormente expuesto en relación con los resultados de otros estudios empíricos y el sistema de género y política. La escasa presencia femenina no puede deberse única ni principalmente a una discriminación sitemática en los partidos políticos. Para poder hablar de discriminación sistemática, tendría que haber un intenso potencial de participación en las mujeres que fuera objeto de represión. Sin embargo, es conocido que, por motivos más bien culturales, las mujeres suelen mostrarse menos participativas, se interesan menos por la política y sienten mayores reparos de abandonar la esfera privada ${ }^{9}$. Sin negar que, por supuesto, existe una discriminación bastante real, la raíz del problema está en la falta de empuje de las mujeres en la sociedad civil y, concretamente, en los partidos. Esto puede ser debido a una falta de preparación psicológica para la competencia, a veces descarnada, que tiene lugar por el poder.

9. Cf. INGLEHART, R. (1991). El cambio cultural en las sociedades industriales avanzadas. Madrid: CIS. 
La batalla para lograr una mayor presencia femenina no está únicamente en el interior de los partidos, sino prioritariamente en los espacios sociales intermedios mencionados en el marco teórico introductorio, que en relación con la política serían la consecución de una posición profesional y educativa como plataforma para el inicio de una carrera política; la corresponsabilidad en las tareas familiares; la conciliación de la vida laboral con la vida familiar para hombres y mujeres y, también, la batalla diaria por la presencia en el seno de los partidos.

Como interrogante se nos plantea la validez de este tipo de estudios. Como resaltan acertadamente E. Uriarte y C.Ruiz ${ }^{10}$, los análisis de listas y resultados electorales no aportan información suficiente para conocer la raíz del problema. La relevancia de este tipo de estudios está limitada a la cuantificación y descripción de este proceso social a través de sus resultados tangibles, por lo tanto la posición y cantidad de mujeres en una lista electoral o en una institución dada es un indicador del grado de integración de la mujer en la sociedad. Sólo por esto, este tipo de estudios ya está justificado. Si bien no se pueden derivar explicaciones de por qué se llega a la discriminación hallada en los datos, sí que se indica dónde hay que buscar las causas: en las prácticas de reclutamiento de los partidos políticos y en los factores ambientales que las condicionan. Para poder llegar a hipótesis explicativas, es necesario ampliar el estudio con encuestas a las afectadas (que en último extremo son todas las mujeres con derecho a sufragio pasivo en un territorio dado) y estudios cualitativos. $\mathrm{Al}$ asumir estas limitaciones, el valor de este estudio queda reafirmado, ya que sus objetivos se limitan al fenómeno en sí, al trabajar con una serie de variables que entendemos como la proyección de unas relaciones de poder que nos vienen dadas. Se aplica un esquema input-output, con una "caja negra» que es el proceso de toma de decisiones individuales y colectivas en relación con la confección de las listas electorales. Pretender interpolar las características concretas de la "caja negra» a partir del output sería injustificable.

Lo que se ofrece en este estudio es una descripción estructural de cómo opera la variable género en función de otras variables dentro de un proceso social de considerable complejidad. La explicación de por qué es así y no de otra manera corresponde a otro estudio. En este sentido, está proyectada por nuestra parte la continuación y complementación de este estudio con los datos correspondientes a 1999, así como la publicación de los resultados de una encuesta dirigida a las mujeres con cargos políticos en Canarias en la legislatura 1995-1999.

10. URIARTE, E.; RUIZ, C. (1999). Mujeres y hombres en las elites políticas españolas. ¿Diferencias o similitudes? REIS, 88, p. 221: «los estudios más recientes [...] aportan [...] nuevos datos que ponen seriamente en cuestión la tesis de la discriminación ejercida por los partidos políticos [...] fundamentada básicamente en el análisis de las características de las listas electorales». 


\section{Bibliografía}

Astelarra, J. (1990). «Las mujeres y la política». En CIS. Participación política de las mujeres. Madrid: Siglo XXI, p. 7-22.

GarCíA de LeÓn, M.A. (1994). Elites discriminadas. Barcelona: Anthropos.

INGLEHART, R. (1991). El cambio cultural en las sociedades industriales avanzadas. Madrid: CIS.

InSTITUTO DE LA Mujer (2000). Resultados electorales. Madrid: Ministerio de Trabajo y Asuntos Sociales.

Molina PetiT, C. (1996). La igualdad no resuelta. Mujer y participación política. Un estudio en Gran Canaria. Las Palmas de Gran Canaria: Instituto Canario de la Mujer.

MONTIEL TORRES, Ana Ma (1998). «Cohesión social y participación femenina». Revista de Fomento Social, núm. 112, vol. 53, p. 502-524.

Romero Navarro, F. (1996). La liberación de la mujer en Gran Canaria. Cambio de la estructura familiar. Las Palmas de Gran Canaria: Universidad de Las Palmas de Gran Canaria. Servicio de Publicaciones.

URIARTE, E.; RUIZ, C. (1999). Mujeres y hombres en las elites politicas españolas. ¿Diferencias o similitudes? REIS, 88, p. 207-232. 